\title{
Brain Imaging Quality Assurance: How to Acquire the Best Brain Images Possible
}

\author{
Barbara J. Grabher, BS, CNMT, RT(N) \\ Grabher Consulting and Specialty Services, Abingdon, Maryland, and Life Molecular Imaging, Inc., Boston, Massachusetts
}

\begin{abstract}
CE credit: For CE credit, you can access the test for this article, as well as additional JNMT CE tests, online at https://www.snmmilearningcenter.org. Complete the test online no later than March 2022. Your online test will be scored immediately. You may make 3 attempts to pass the test and must answer $80 \%$ of the questions correctly to receive $1.0 \mathrm{CEH}$ (Continuing Education Hour) credit. SNMMI members will have their CEH credit added to their VOICE transcript automatically; nonmembers will be able to print out a CE certificate upon successfully completing the test. The online test is free to SNMMI members; nonmembers must pay $\$ 15.00$ by credit card when logging onto the website to take the test.
\end{abstract}

Multiple factors affect image quality regardless of the organ being imaged. Every aspect of an image protocol must be followed to ensure the highest image quality and to prevent misinterpretation of the findings and an incorrect final report, which could negatively affect patient management. It is important that nuclear medicine technologists understand what can affect brain image quality and learn how to overcome challenges in performing any type of brain imaging, be it SPECT, dopamine transporter imaging, amyloid imaging, or ${ }^{18}$ F-FDG PET.

Key Words: brain imaging; image quality; patient positioning; technical; quality assurance

J Nucl Med Technol 2019; 47:13-20

DOI: 10.2967/jnmt.118.211771

$\mathbf{O}$ ptimal imaging of the brain using molecular imaging techniques relies on multiple factors. The aim of this continuing education article is to review the administrative, technical, and interpretative factors that can affect image quality when acquiring any type of nuclear medicine brain image. The article will help the nuclear medicine technologist (NMT) understand the relationship between proper patient positioning and image quality regardless of the type of brain imaging being performed. These techniques are applicable to both SPECT and PET. Examples are provided for dopamine transporter (DaT) imaging, amyloid imaging, and brain ${ }^{18} \mathrm{~F}$-FDG PET; however, the positioning techniques in this article can also be applied to perfusion imaging with ${ }^{99 \mathrm{~m}} \mathrm{Tc}$ tracers. The article also illustrates the importance of following the instructions in a radiopharmaceutical package insert, including acquisition and processing parameters, to maximize image quality. Lastly, this article reviews how to make

Received Sep. 11, 2018; revision accepted Nov. 13, 2018.

For correspondence or reprints contact: Barbara J. Grabher, Grabher Consulting and Specialty Services, 3113 Pouska Rd., Abingdon, MD 21009. E-mail: barbara.grabher@gmail.com

Published online Jan. 25, 2019.

COPYRIGHT (C) 2019 by the Society of Nuclear Medicine and Molecular Imaging. postacquisition adjustments to improve image quality when needed. A recent report (I) stated that the compounded annualized growth rate for molecular imaging is expected to be $7.5 \%$ between 2017 and 2027. This growth rate is being fueled by the use of cutting-edge technology such as PET/ $\mathrm{CT}$ and PET/MR and by the new molecular imaging radiotracers being introduced into the market for such uses as movement-disorder imaging, amyloid plaque imaging, and prostate imaging. It is imperative that NMTs and interpreting physicians understand all factors that can affect the quality of brain imaging and how to improve scan quality when challenges arise.

\section{DEFINITION OF QUALITY}

Quality can be defined and measured in different ways depending on the industry involved. In manufacturing, quality is defined as "A measure of excellence or a state of being free from defects, deficiencies and significant variations" (2). Many manufacturing companies, such as Toyota, General Electric, and Motorola, use Six Sigma and Lean Six Sigma process improvement practices and techniques to help measure and improve quality in the products they manufacture and the services they provide. A Six Sigma level is defined as 3.4 defects per 1 million opportunities (3). Most average companies operate at 3 or 4 sigma, whereas world-class companies operate at 5 or 6 sigma (4). An efficiency level of 99.9\% might seem excellent, but from a Six Sigma perspective, $99.9 \%$ efficiency equates to the following (4):

- 50 newborn babies per day dropped at birth by doctors

- $10 \mathrm{~h}$ of unsafe drinking water produced every month

- Two unsafe plane landings per day at O'Hare, or 4,380 per year

- 16,000 pieces of mail per hour lost by the post office, or over 140 million per year

- 500 incorrect surgical operations per week, or 26,000 per year

- 22,000 checks per hour deducted from the wrong bank account, or over 192 million per year

- 32,000 missed heartbeats per person per year 
TABLE 1

Examples of Health-Care Organizations That Implemented Lean Six Sigma Projects (17)

\begin{tabular}{|c|c|c|}
\hline Hospital & Project & Effect \\
\hline $\begin{array}{l}\text { Boston Medical } \\
\text { Center }\end{array}$ & $\begin{array}{r}\text { Project to emphasize } \\
\text { diagnostic imaging }\end{array}$ & $\begin{array}{l}\text { Cost savings and revenue increases of } \\
\text { more than } \$ 2.2 \text { million }\end{array}$ \\
\hline $\begin{array}{l}\text { Rapides Regional } \\
\text { Medical Center }\end{array}$ & $\begin{array}{l}\text { Project to increase } \\
\text { efficiency of emergency department }\end{array}$ & $\begin{array}{l}\text { Drop in wait times; increase in patients seen; } \\
\text { annual savings of more than } \$ 950,000\end{array}$ \\
\hline $\begin{array}{l}\text { Valley Baptist } \\
\text { Health System }\end{array}$ & $\begin{array}{l}\text { Project to reduce surgery } \\
\text { cycle time }\end{array}$ & $\begin{array}{l}\text { Increase in annual capacity by } 1,100 \\
\text { more cases, for potential income of } \$ 1.3 \text { million }\end{array}$ \\
\hline $\begin{array}{l}\text { Yale-New Haven } \\
\text { Medical Center }\end{array}$ & $\begin{array}{l}\text { Multiple projects in surgical } \\
\text { intensive care unit }\end{array}$ & $\begin{array}{l}75 \% \text { decrease in bloodstream infections, } \\
\text { for annual savings of } \sim \$ 1.2 \text { million }\end{array}$ \\
\hline
\end{tabular}

These examples show that $99.9 \%$ is not as good as may be perceived and that there is always room for improvement in any industry. Many Six Sigma experts have estimated that a company operating at between 3 and 4 sigma can expect about a $10 \%$ loss in revenues from inefficiency (5). In today's competitive business environment, no company, hospital, or nuclear medicine department can afford to lose revenue because of inefficiency.

In health care, quality is measured and described in many ways. According to Carolyn M. Clancy, who provided testimony before the Committee on Finance, Subcommittee on Health Care, United States Senate, in March 2009: "Health care quality is getting the right care to the right patient at the right time-every time" (6). Failure in reaching this goal costs organizations money. Additional labor, additional materials, and dissatisfaction on the part of both the patient and the physician result in loss of revenue, which takes away from the hospital's profitability and the overall success of the health-care system. In the past decade, many health-care organizations have started using Six Sigma and Lean Six Sigma practices and procedures to help ensure the highest-quality and most efficient care, as well as continued success and profitability (4). Six Sigma methodologies are used to analyze and streamline routine processes and procedures to improve every aspect of their quality. Table 1 provides some examples of Six Sigma projects that improved the profitability of the health-care organizations that implemented them. Table 2 provides some examples of projects that could be implemented in a nuclear medicine or radiology department to improve efficiency and quality. Other examples of Six Sigma projects include reduction of noise at night, reduction of hospital readmissions, reduction of cancellations, improvement of pain management, improvement of call-bell response time, improvement of the discharge process, improvement of medication education, improvement of patient flow, and improvement of rehab-patient satisfaction. All such projects lead to improved quality of care for patients and a stronger, more financially secure health-care organization.

\section{THREE AREAS OF QUALITY IN IMAGING}

There are 3 general areas of quality in imaging: administrative, technical, and interpretative (Fig. 1) (5). Each of these can have an effect on the others. One can do everything right regarding the administrative and technical quality of a scan; but if the interpretative quality is not up to par, the final report and overall quality of the scan could be suboptimal. Suboptimal reporting can potentially result in missing information or in communication of misleading information to the referring physician. Even worse, the scan could be misread, potentially leading to a wrong diagnosis. It is essential that all areas of image quality receive attention to ensure that the highest-quality scan and results are provided to the referring physician.

\section{ACQUISITION OF GOOD-QUALITY BRAIN IMAGES}

The first step in acquiring a good brain image is to follow all the administrative, technical, and interpretative guidelines for the particular protocol and to follow the prescribing information in the radiopharmaceutical package insert. For example, if the package insert says to inject at $6 \mathrm{~s}$ per milliliter, the radiopharmaceutical must be injected

TABLE 2

Examples of Lean Six Sigma Projects for Radiology or Nuclear Medicine Departments

\begin{tabular}{cc}
\hline \multicolumn{1}{c}{ Project } & Purpose \\
\hline $\begin{array}{c}\text { Analysis of report generation times (time physician ordered } \\
\text { study to time physician received final report) }\end{array}$ & Decrease waiting time to receive reports \\
$\begin{array}{c}\text { Analysis of patient escort times to and from nuclear medicine } \\
\text { department }\end{array}$ & $\begin{array}{c}\text { Ensure proper staffing of escort department; increase } \\
\text { on-time patient arrivals; increase number of patients } \\
\text { scanned }\end{array}$ \\
& $\begin{array}{c}\text { Decrease waste and improve efficiency in imaging } \\
\text { department }\end{array}$ \\
\hline
\end{tabular}




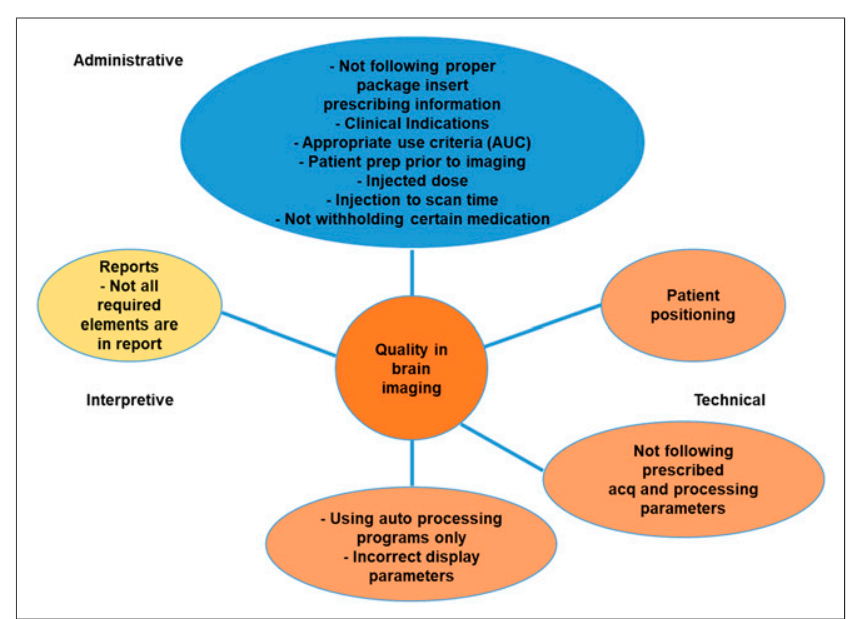

FIGURE 1. Three areas of quality in brain imaging: administrative, technical, and interpretative.

at that rate. Instructions in a package insert are there for a reason and should be followed for best results. However, there is one more thing NMTs can do to ensure the best brain images possible regardless of type of procedure: technical consistency. For the best image quality, technical consistency is needed in patient preparation, patient positioning, acquisition parameters, reconstruction parameters, orientation of amyloid and ${ }^{18} \mathrm{~F}-\mathrm{FDG}$ brain slices (transverse, sagittal, and coronal), orientation of ${ }^{123}$ I-ioflupane (DaTscan; GE Healthcare) images (especially coronal tilt), and display parameters. It is also important for the NMT to avoid overprocessing, making images look too smooth and possibly causing the findings to be misinterpreted. Not being technically consistent in how patients are positioned or images processed may negatively affect the interpretation of the scan. If individual NMTs change processing parameters on the basis of their eye, the physician may get inconsistent results depending on which NMT did the processing. Being technically consistent in all aspects of an imaging protocol is key to achieving the highest-quality scan and highest-quality report. According to Sajdak et al., following standard operating procedures helps ensure that nuclear medicine physicians and NMTs follow an imaging protocol helping to ensure reproducibility and consistency, which in turn are key in producing the highest-quality images (7).

\section{PATIENT POSITIONING}

Patient positioning is a critical technical step in the imaging protocol. Proper positioning of the patient reduces the likelihood of artifacts due to motion, promotes patient comfort, and, most importantly, helps interpretation by providing standardized and artifact-free scans. The NMT should take the extra time to thoroughly explain the procedure to the patient and ensure that the patient is comfortable and secure on the table so there is less chance the patient will move and the scan will need to be repeated.

Before any type of brain scan, patients should use the restroom and remove their eyeglasses, earrings, hair clips or combs, and hearing aids. The patient's head should be in a head holder, with the vertex of the head at the superior edge of the head holder (Fig. 2). If the head holder has extended sides, foam wedges, folded sheets, or blue chux (absorbent pad) should be placed between the head and the head holder to stabilize the head. Feeling these materials against their face can also be a reminder for patients to hold their head still during the scan.

The canthomeatal line is another landmark or reference point that helps position the patient's head properly in the head holder. The canthomeatal line is an imaginary vertical line drawn from the external canthus (external corner) of the eye to the meatus (center) of the ear. It is important to make sure that the canthomeatal line is vertical and perpendicular to the imaging table. Figure 2 shows where the canthomeatal line is located. If for some reason the line cannot be positioned vertically and perpendicular to the imaging table, the patient's head should be in a natural, relaxed position and secured with a chin strap and a head strap to prevent movement. The brain can then be reoriented into its proper position when the images are processed. As reported by Covington et al. (8), "Even small deflections of the canthomeatal line from the vertical position may signify abnormal head tilt."

Once properly positioned in the head holder, the patient's head is secured using self-adherent chin and head wraps (cohesive tape) to prevent movement. These should be used even if patients say they can remain still. Patients' arms are positioned at their sides and stabilized using bed straps or other means to minimize movement. If placed on the stomach without a body strap, a patient's arms may tire and begin moving, which in turn could cause the shoulders to move and possibly the neck and head. A foam wedge or pillow is placed under the patient's knees, and the patient is covered with a blanket to ensure warmth and comfort. If the scanner is equipped with lasers, they can be used to properly position the patient's head and adjust the table height so that the head is in the center of the field of view. All brain imaging

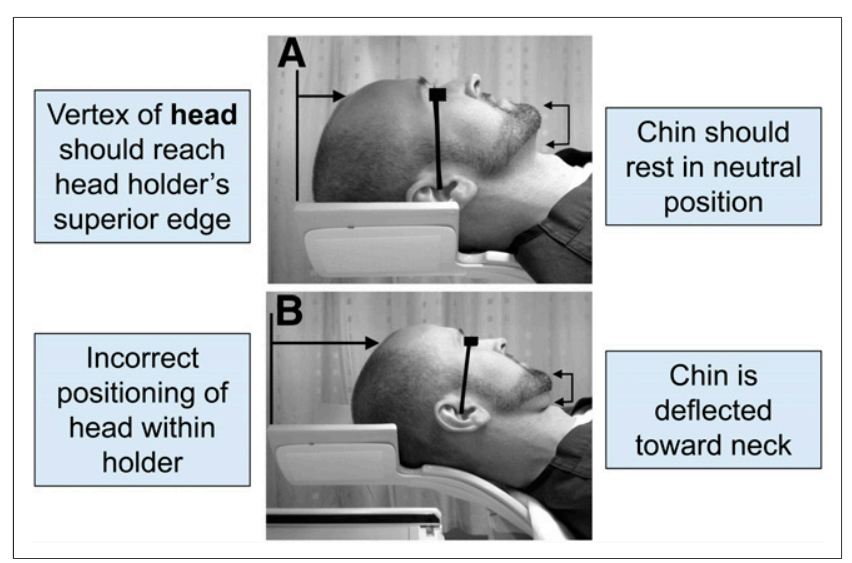

FIGURE 2. (A) Proper positioning in head holder. Canthomeatal line is imaginary line that runs from lateral corner of eye to ear canal. (B) Improper positioning. Canthomeatal line is not vertical and perpendicular to imaging table. (Reprinted from (8).) 


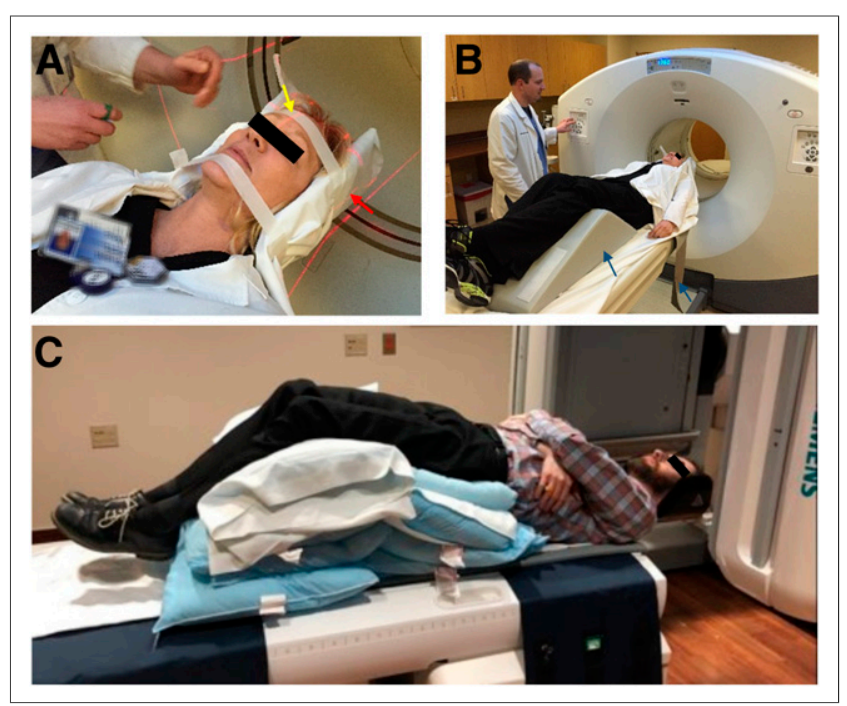

FIGURE 3. (A) Proper positioning on imaging table using head strap, chin strap, and laser lights. Laser light denoted by yellow arrow is positioned at center of nose and forehead to prevent head rotation. Laser light denoted by red arrow, at level of ear, positions table height so that head is in middle of field of view. (Courtesy of Virtua Health, Voorhees, NJ.) (B) Knee cushion and body strap denoted by arrows. (Courtesy of Virtua Health, Voorhees, NJ.) (C) Challenge of positioning patients with kyphosis, severe neck pain, or severe back pain. Multiple pillows under legs, buttocks, and lower back help patient lie flat. Use of body straps to secure patient to table is important.

requires that the patient's brain be in the center of the field of view, with complete coverage of the cerebellum, especially for amyloid or ${ }^{18}$ F-FDG brain imaging. Figure 3 shows images of proper patient positioning on the imaging table using the head strap, chin strap, body strap, knee cushion, and laser light system. The last step before the scan is to view the head position from the end of the scanning table and correct for any signs of head rotation.

Not all patients can lie flat and still on the imaging table, such as those with severe arthritis, back issues, neck issues, or kyphosis. Positioning these types of patients can be challenging. However, with a little coaching, support, and a few pillows, even these patients can be positioned in such a way that the best images possible are achieved. Figure 3C illustrates the best way to position patients with kyphosis or severe neck and back pain, a positioning trick I learned many years ago from an x-ray technologist. It looks a little scary, and it appears as if one is dumping the patient on the head, but it works every time. As long as the patient is comfortable and strapped securely to the table, such positioning tricks can be used for all kinds of brain imaging.

Another patient positioning challenge pertains to DaTscan imaging. Although a patient may be able to lie flat and be still for the entire 30-min scan, the distance of the camera head from the patient is also critical. The resolution of an image decreases as the camera distance increases (9), and positioning the camera head as close as possible is key to goodquality DaTscan images. The package insert recommends that the camera radius be between 11 and $15 \mathrm{~cm}$ (10). This is especially important in DaTscan imaging because the caudate and putamen are small structures about the size of a cashew in the middle of the brain (Fig. 4) (11). In imaging two such structures using a large-field-of-view camera with camera heads approximately $91 \mathrm{~cm}$ (36 in) long and $61 \mathrm{~cm}$ (24 in) wide, one can see why the camera radius must be as close as possible. Figure 4C illustrates what happens to image quality when the camera radius increases from the recommended $12.8 \mathrm{~cm}$ to $15.0 \mathrm{~cm}$ and $20.0 \mathrm{~cm}$. As the radius increases, the putamen or tails of the image appear shorter, potentially causing the physician to interpret the findings as positive when in reality the findings are negative based on the 12.8-cm image. Figure 4D illustrates how close the camera head should be to the patient. Achieving this is not easy. Patients may be afraid the camera will hit them, and NMTs, especially if inexperienced with DaTscan imaging, might feel nervous about moving the camera heads so close to the patient. Practicing positioning with nuclear medicine colleagues

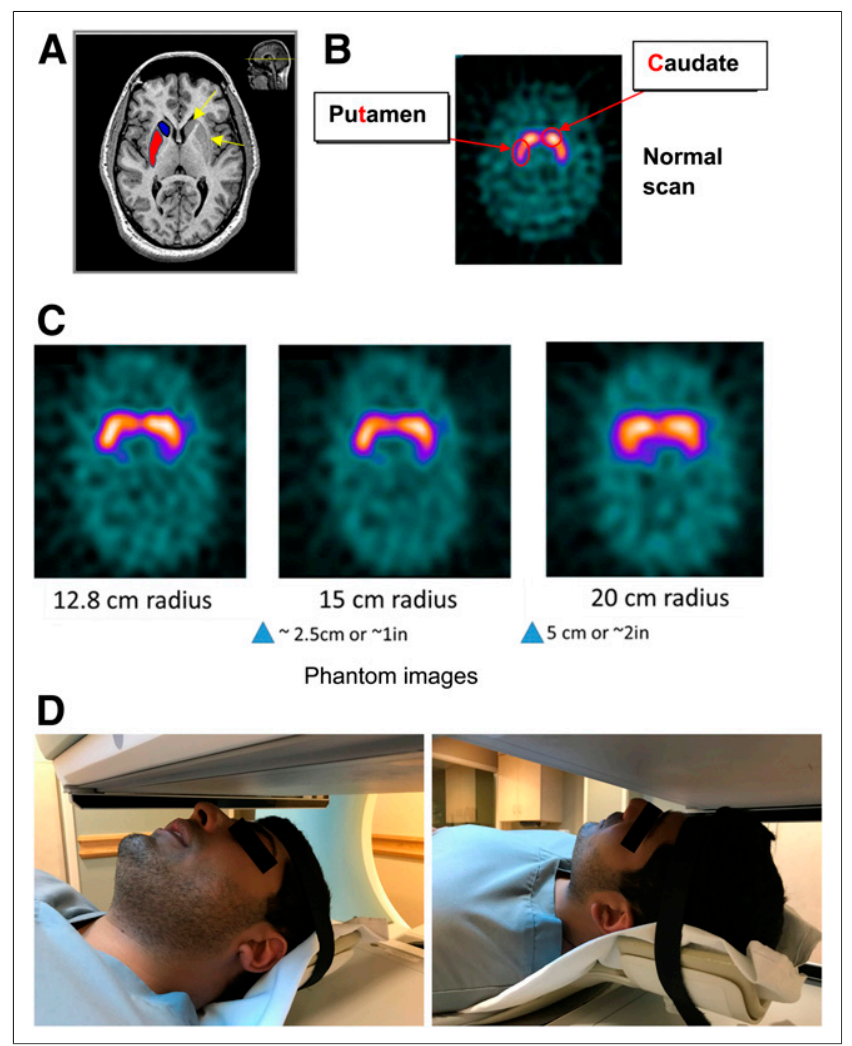

FIGURE 4. Key DaTscan positioning considerations. (A) Location of caudate nucleus and putamen within brain on MRI. Blue denotes right caudate, red denotes right putamen, and arrows denote left caudate and left putamen. (B) Transverse DaTscan image of caudate and putamen. (Courtesy of Holy Name Hospital, Teaneck, NJ.) (C) Effect of increasing camera radius on DaTscan image quality. As radius increases, quality decreases. Putamen looks shorter and less defined with $20-\mathrm{cm}$ radius than with $12.8-\mathrm{cm}$ radius. (Courtesy of Liz Clarke.) (D) Camera head must be as close as possible to nose for best image quality and must clear shoulders. (Courtesy of Holy Name Hospital, Teaneck, NJ.) 


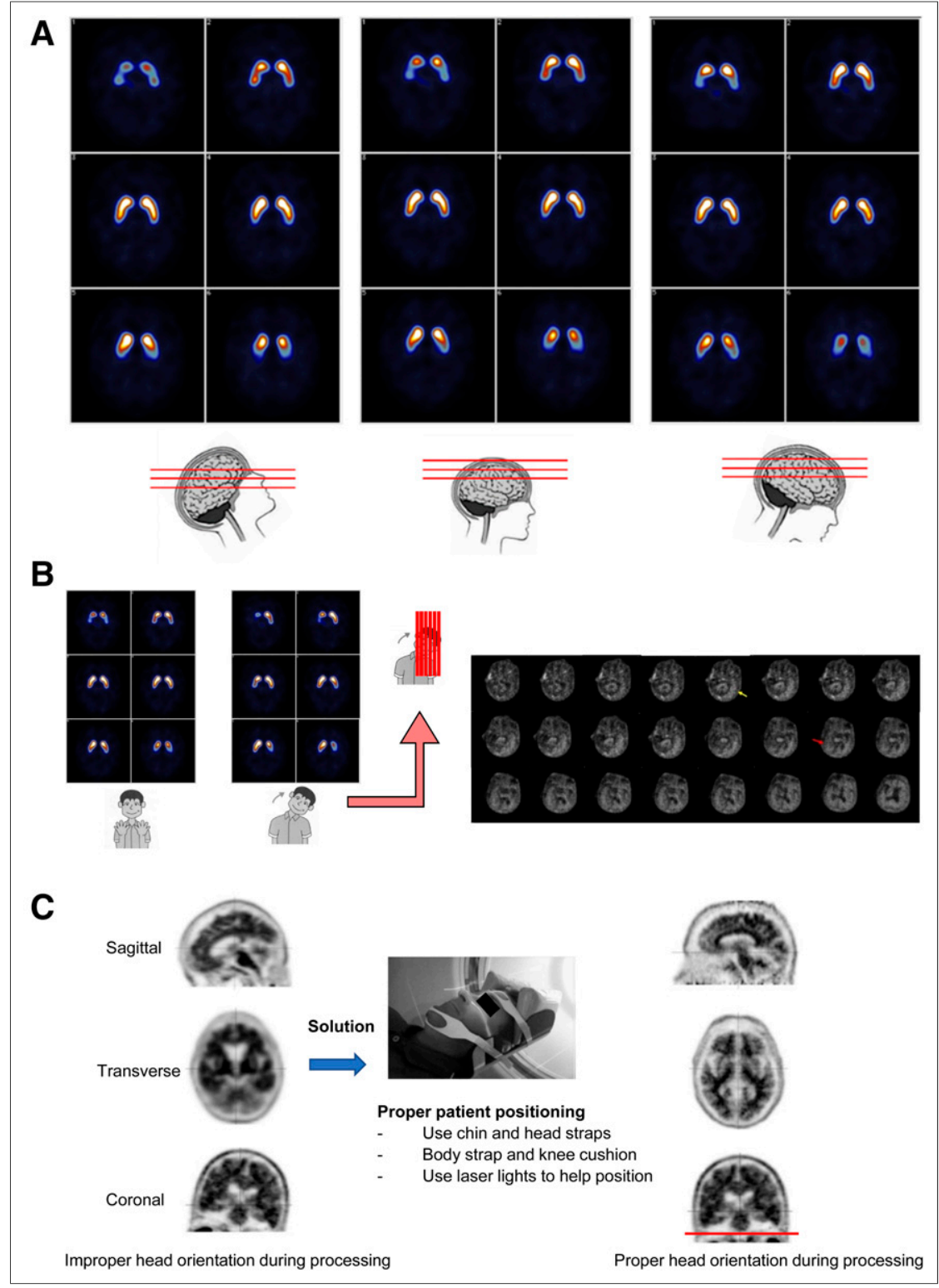

FIGURE 5. Effect of head orientation on DaTscan imaging. (A) Forward, backward, or sagittal head tilt has no effect on image quality. (Courtesy of Glasgow Southern Hospital.) (B) Lateral head tilt shows one putamen before the other, complicating interpretation. On amyloid imaging (right), yellow arrow denotes image on which left lateral lobe is first seen, and red arrow denotes image on which right lateral temporal lobe is first seen, almost 10 frames later. (Courtesy of Piramal Imaging.) (C) Head is tilted too far forward in sagittal slice at left and too far right in coronal slice. Although head position can be corrected during processing, best solution is to correctly position patient prior to image acquisition. Red line shows positioning that will result in properly oriented images after processing.

will increase the confidence of the NMT and therefore the comfort of the patient. Shoulder clearance is also an important factor to consider in setting the radius.

\section{HEAD ORIENTATION}

Head orientation is another important aspect of patient positioning in all brain imaging. If head orientation is incorrect or not fixed manually during processing, displayed images may be difficult to interpret, possibly reducing image quality and affecting the final report. Sagittal tilt (forward or backward head tilt) has little effect on image quality in a DaTscan (Fig. 5A) but can be a factor in an amyloid or ${ }^{18} \mathrm{~F}-\mathrm{FDG}$ scan. With a forward head tilt on amyloid imaging, the frontal lobes will be seen before the lateral temporal lobes appear, and with a backward head tilt the cerebellum and parietal lobes will be seen before the lateral temporal lobes appear. Unfortunately, lateral tilt can cause difficulties in both DaTscan and amyloid imaging (Fig. 5B). There are several ways to improve image quality related to head orientation. First, proper positioning can be ensured at the beginning of the scan, making use of laser lights to aid in orienting the head and adjusting the table height. Second, the processing angles can be manually adjusted to reorient the brain, correcting for sagittal and coronal head tilt. It is best to use the coronal image to check for head tilt. Figure 5C illustrates properly and improperly positioned images and a solution to achieving a better orientation. Improperly positioned images during processing could lead the physician to misinterpret the findings on the final images. Third, the interpreting physician can be told that positioning the patient was challenging and out of the norm and that the orientation of the patient's head during the processing may be affected.

\section{PROCESSING AND DISPLAY PARAMETERS}

If not followed properly, the processing and display parameters can also affect image quality. Positioning of processing lines is a critical step in reconstruction in all types of brain imaging and can affect the final appearance. Proper reconstruction is based on the intercommissural line, which passes through the center of the anterior commissure (AC) and the posterior commissure (PC). The AC-PC line passes through the superior surface of the AC and the center of the PC. Figure 6 illustrates where these lines are located. They are commonly used to help consistently position patients' brains during processing of brain imaging, be it nuclear medicine (both SPECT and PET), MRI, or CT (12).

Incorrectly positioning these lines when processing any brain scan can be an issue. With ${ }^{18}$ F-FDG or amyloid imaging, 


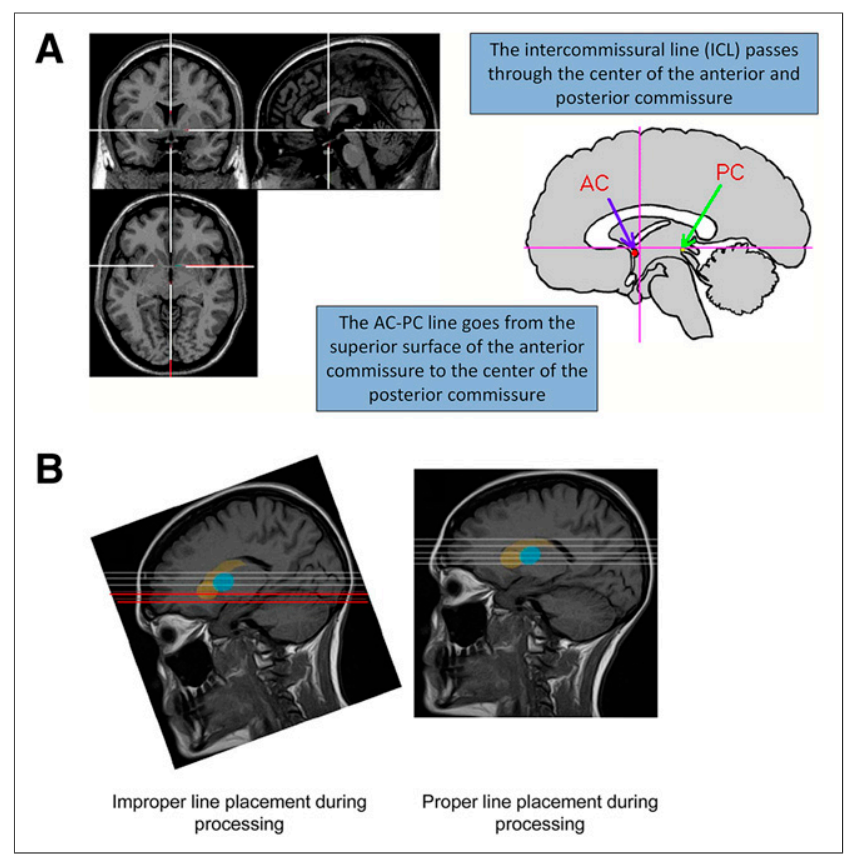

FIGURE 6. (A) Location of intercommissural line and AC-PC line. (B) MRI study showing improper and proper placement of processing lines. Forward head tilt results in slices (red lines) through caudate head that do not include putamen. On DaTscan studies, false-positive findings can result from incorrect positioning of processing lines. (Reprinted from (8).)

incorrect line placement can result in some regions of the brain being seen before others, such as the frontal lobes before the lateral temporal lobes. If lateral head tilt is not adjusted during processing, poor image quality can also result (Fig. 5B). Special care needs to be taken when positioning the processing lines for a DaTscan study, as incorrect positioning can create false-positive findings (Fig. 6B).

Another component of image processing that can affect image quality is the filter setting and whether the images are processed using filtered backprojection or iterative reconstruction. In amyloid imaging, it is usually fine to use the scanner's default filter settings for the brain protocol, but the filters can be changed if the physician feels the images need to be smoother or have more contrast. Once a setting is chosen, it should be used consistently and not changed. DaTscan filter settings also need to be chosen properly; selecting a cutoff that is too low-say, 0.2-will make the images look too smooth, and selecting a cutoff that is too high - say, 0.6-will remove too many counts and make the image look too grainy. Figure 7 illustrates what a DaTscan study looks like with different filter settings. A good cutoff is $0.4-0.5$, depending on the preference of the interpreting physician for the particular SPECT camera being used.

The color display used for brain images can also influence image quality. The prescribing information for each radiopharmaceutical will usually recommend a particular color display to use when interpreting images. If no recommendation is given about the color scale, the interpreting physician should be consistent with whatever color scale is chosen. Using a color scale that does not provide a sufficient color gradient within the image could make a physician overread a scan.

\section{CONSIDERATIONS AND ADJUSTMENTS}

As health-care professionals, we want to provide patients with optimal quality throughout the procedure, but sometimes issues occur that can negatively affect a patient's experience or image quality. The camera may go down, or the patient or dose may arrive late. The NMT should be able to recognize potential sources of artifact-causing errors and learn how to correct them. The NMT should also tell the physician of any unusual issues that arise and discuss how to proceed. An example is a patient who arrives late, so that the dose has dropped to slightly below the prescribed range. Technically, the dose should not be injected, but canceling the scan would cause an expensive PET radiopharmaceutical to be wasted. If, on discussion, the nuclear medicine physician decides to proceed with the scan anyway, the NMT would then have to know to increase the scan time slightly to ensure enough counts are acquired to get good-quality images.

Another consideration is that NMTs should be willing to help with tasks that are not part of their usual responsibilities, or to find someone who can help. Maybe a less experienced technologist is doing something wrong while processing a brain scan. A senior technologist who notices can jump in and use the occasion as a teaching moment. NMTs should always strive to do the right thing for the patient and ensure that doses are optimized to help eliminate unnecessary scans.

\section{IMAGE INTERPRETATION AND REPORTING}

Image interpretation and reporting is the final step in the quest for the best-quality brain images possible. NMTs can do everything right on their end in positioning the patient and acquiring and displaying the best images, but if the interpreting physician misreads something or leaves something out of a report, the quality of the scan will be affected. Interpreting physicians should review the radiopharmaceutical package

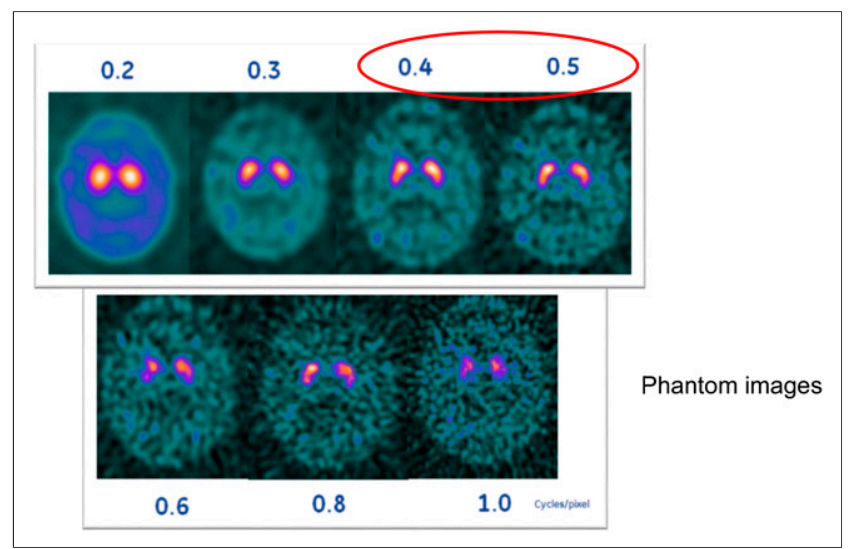

FIGURE 7. DaTscan study with different filter settings. Cutoff of $0.4-0.5$ is usually good, depending of type of SPECT camera. Once interpreting physician chooses filter setting, it should be applied consistently for each study. (Courtesy of Liz Clarke.) 
insert information, the equipment and software manufacturers' instructions, and professional organizations' recommendations as related to the images they are interpreting, as well as obtain any necessary required training (e.g., amyloid imaging). If technologists or physicians have any questions about optimizing camera settings or using the radiopharmaceutical, they should reach out to the camera manufacturer, clinical applications specialists, or radiopharmaceutical manufacturer.

Another important step during the interpretation process is to make sure the interpreting physician reads images in the appropriate color scale, if required. Ensuring that the dictated report has all the necessary components is also important. For amyloid imaging, these reporting components can be found in a document from the Society of Nuclear Medicine and Molecular Imaging (SNMMI) and the European Association of Nuclear Medicine (13). Another document helpful for interpreting physicians, ordering physicians, and NMTs is the appropriate use criteria for amyloid PET (14). Both documents provide useful information on all aspects of amyloid imaging, ensuring the highest-quality amyloid images possible if followed properly. Information on ${ }^{18} \mathrm{~F}-\mathrm{FDG}$ and DaTscan imaging is provided in 2 guidelines from the SNMMI $(15,16)$. It is important for the nuclear medicine physician to report any abnormal finding to the neurologist or ordering physician as soon as possible.

\section{CHECKLIST TO ACHIEVE OPTIMAL BRAIN IMAGES}

The best way for NMTs and interpreting physicians to make sure they are achieving optimal brain images every time is to practice consistency in everything they do and maintain good communication. Using a checklist such as the one below will help with consistency:

- Follow all instructions in the package insert for the radiopharmaceutical being used.

- Explain to the patient the importance of following instructions and being still.

- Tell the patient to empty the bladder immediately before imaging.

- Position the patient comfortably on the table, with the head comfortably in the head holder. Take extra time, if needed, to make sure the patient is as comfortable as possible on the table.

- Secure the head using the head strap and chin strap, regardless of whether the patient thinks it necessary.

- Use positioning lasers to make sure the head is straight and in the field of view.

- For SPECT images, position the camera radius as close as possible to the patient's nose $(11-15 \mathrm{~cm})$, and check that the shoulders are cleared.

- During the acquisition, stay with the patient to watch for movement.

- Review the raw data images for movement before allowing the patient to leave.

- Review the reconstructed images for movement; do not rely on auto processing.
- If the head looks tilted, manually adjust the processing lines using the AC-PC line.

- Use the correct filter cutoff and display settings.

- Tell the interpreting physician if anything out of the ordinary happened during the positioning of the patient or the acquisition of the scan.

\section{CONCLUSION}

Nuclear medicine brain imaging is being used increasingly across the country to evaluate many different conditions, including amyloid plaque, movement disorders such as essential tremor and Parkinson disease, and other neurologic disorders. Many factors can affect the quality of brain images. Following the appropriate imaging technique and the package insert is necessary to achieve optimal image quality and accurate and reliable image results. Patient positioning, particularly the head position, is critical in acquiring any goodquality brain image, and in DaTscan imaging the camera radius is also critical. Technical consistency is required in every aspect of a patient's scan. Each area of quality - administrative, technical, and interpretative - can influence the others and affect the images, their interpretation, and the subsequent treatment and outcome of the patient. NMTs have a huge role in ensuring image quality. Making it personal-performing every brain scan as if on a family member-may help NMTs achieve the level of health-care quality defined by Dr. Clancy in her testimony to Congress, that of "getting the right care to the right patient at the right time-every time" (6).

\section{DISCLOSURE}

Barbara Grabher is chief executive officer of Grabher Consulting and Specialty Services and an employee of Life Molecular Imaging, Inc. (formerly known as Piramal Imaging), Boston, MA. No other potential conflict of interest relevant to this article was reported.

\section{ACKNOWLEDGMENT}

I thank Mary Beth Farrell for her editorial assistance with this article. The information outlined in this article was presented at the SNMMI annual meeting held in Philadelphia, Pennsylvania, on June 23, 2018.

\section{REFERENCES}

1. Molecular imaging market to be worth US\$ 6,445.8 $\mathrm{mn}$ by 2027 with CAGR $7.5 \%$ : future market insights. GlobeNewswire website. https://globenewswire.com/newsrelease/2017/12/20/1266788/0/en/Molecular-Imaging-Market-to-be-worth-US-6-4458-Mn-by-2027-with-CAGR-7-5-Future-Market-Insights.html. Published December 20, 2017. Accessed January 8, 2019.

2. Quality. Business Directory website. http://www.businessdictionary.com/definition/ quality.html. Accessed January 8, 2019.

3. Save your company a fortune: ASQ Six Sigma business solutions. ASQ website. http://asq.org/public/six-sigma-training/asqsigma.pdf. Published 2009. Accessed January 8, 2019.

4. Lean Six Sigma: the basics. Chi Solutions website. https://www.chisolutionsinc. com/wp-content/uploads/2015/01/2008_10_Lean-Newsletter-Article.pdf. Published October 2008. Accessed January 8, 2019. 
5. Evans MH. Reaching for Six Sigma. Excellence in Financial Management website. http://www.exinfm.com/board/reaching_for_six_sigma.htm. Accessed on April 26, 2018.

6. What is health care quality and who decides? U.S. Senate Committee on Finance website. https://www.finance.senate.gov/imo/media/doc/640431.pdf. Published March 18, 2009. Accessed January 8, 2019.

7. Sajdak R, Trembath L, Thomas K. The importance of standard operating procedures in clinical trials. J Nucl Med Technol. 2013;41:231-233.

8. Covington MF, McMillan NA, Avery RJ, Kuo PH. The semicolon sign: dopamine transporter imaging artifact from head tilt. J Nucl Med Technol. 2013;41: 105-107.

9. Rosenthal MS, Cullom J, Hawkins W, Moore SC, Tsui BM, Yester M. Quantitative SPECT imaging: A review and recommendations by the Focus Committee of the Society of Nuclear Medicine Computer and Instrumentation Council. J Nucl Med. 1995;36:1489-1515.

10. DaTscan [prescribing information]. Arlington Heights, IL: GE Healthcare; 2015.

11. Lanciego JL, Luquin N, Obeso JA. Functional neuroanatomy of the basal ganglia. Cold Spring Harb Perspect Med. 2012;2:a009621.

12. Trembath A, Opanowski A. PET imaging of the brain for technologists: CTN webinar series 2016. IDEAS-Study website. https://www.ideas-study.org/wp-
content/uploads/2017/01/PET-Imaging-of-the-Brain-for-Technologists_04Apr16.pdf. Published 2016. Accessed January 8, 2019.

13. Minoshima S, Drzezga AE, Barthel H, et al. SNMMI procedure standard/EANM practice guideline for amyloid PET imaging of the brain 1.0. J Nucl Med. 2016;57: 1316-1322.

14. Johnson KA, Minoshima S, Bohnen NI, et al. Appropriate use criteria for amyloid PET: a report of the Amyloid Imaging Task Force, the Society of Nuclear Medicine and Molecular Imaging, and the Alzheimer's Association. J Nucl Med. 2013;54:476-490.

15. Djang DS, Janssen MJ, Bohnen N, et al. SNM practice guideline for dopamine transporter imaging with ${ }^{123}$ I-ioflupane SPECT 1.0. J Nucl Med. 2012;53:154-163.

16. Waxman A, Herholz K, Lewis DA, et al. Society of Nuclear Medicine Procedure Guideline for FDG PET brain imaging: version 1.0. SNMMI website. http:// snmmi.files.cms-plus.com/docs/Society\%20of\%20Nuclear\%20Medicine $\%$ 20Procedure\%20Guideline\%20for\%20FDG\%20PET\%20Brain\%20Imaging.pdf. Published February 8, 2009. Accessed January 8, 2019.

17. Mehrotra S. Lean Six Sigma methodologies: How do they improve health care industry? GreyCampus website. https://www.greycampus.com/blog/ quality-management/health-care-industry-can-reduce-wastage-by-these-leansix-sigma-methodologies. Published December 1, 2017. Accessed January 8, 2019 